\title{
Características clínicas y funcionales según género de pacientes con enfermedad pulmonar obstructiva crónica
}

\author{
JORGE DREYSE D.**, CARMEN LISBOA B.*, CLAUDIO PINTO R.**, \\ FERNANDO SALDÍAS P.* y ORLANDO DÍAZ P.*
}

\section{Clinical and functional characteristics of COPD according to gender}

Chronic obstructive pulmonary disease (COPD) prevalence is continuously rising in females. In addition, mortality due to COPD is higher in females, suggesting that women may develop a more severe disease than males. Our aim was to study possible differences in disease severity according to gender in 95 COPD patients (38 females) consecutively recruited to participate in a follow up protocol. Severity of COPD was assessed by FEV,$B O D E$ index and the impairment of quality of life was measured with the Saint George's respiratory questionnaire (SGRQ) and the chronic respiratory questionnaire (CRQ). We found no differences according to gender in any of the severity indices studied. However, female smoked significantly less than males (35.5 \pm 19.4 versus $45.7 \pm 21$ pack-year; $p=0.02)$. Our results suggest that women are more susceptible to develop COPD than men, without differences in disease severity.

Key words: $C O P D$, lung function, BODE index, gender.

\section{Resumen}

La prevalencia, al igual que la mortalidad de la enfermedad pulmonar obstructiva crónica (EPOC), ha aumentado en las mujeres. Esto último sugiere que la enfermedad sería más grave en el sexo femenino. El propósito de este estudio fue evaluar si existen diferencias en la gravedad según género en 95 pacientes (38 mujeres) con EPOC ingresados consecutivamente a un protocolo de seguimiento. Evaluamos la magnitud del tabaquismo, características demográficas, gravedad según $V E F_{1}$ e índice BODE y compromiso de la calidad de vida. Los resultados no demostraron diferencias entre hombres y mujeres en ninguno de los índices de gravedad. Sin embargo, la magnitud del tabaquismo fue inferior en las mujeres que en los hombres (35,5 $\pm 19,4$ vs 45,7 \pm 21 paquetes-año; $p=0,02)$. Nuestros resultados sugieren una mayor susceptibilidad de las mujeres para desarrollar EPOC, pero no demuestran que la gravedad de la enfermedad dependa del género.

Palabras clave: EPOC, función pulmonar, índice BODE, género.

\section{Introducción}

La enfermedad pulmonar obstructiva crónica (EPOC) ha sido considerada por años como una enfermedad que ocurre preferentemente en hombres, lo que se ha atribuido en parte al mayor nivel de tabaquismo en ellos ${ }^{1} \mathrm{y}$ al hecho de que los médicos son menos proclives a diag- nosticar EPOC en las mujeres ${ }^{2}$. Sin embargo, diversas observaciones demuestran que la EPOC ha aumentado progresivamente en las mujeres en varios países ${ }^{3-5}$ y que su mortalidad en el año 2000 superó a la de los hombres ${ }^{3}$. Esta situación podría explicarse por el aumento del consumo de tabaco en las mujeres y por una posible mayor susceptibilidad a desarrollar EPOC,

\footnotetext{
* Departamento de Enfermedades Respiratorias, Pontificia Universidad Católica de Chile.

** Ayudante alumno de la Escuela de Medicina, Pontificia Universidad Católica de Chile.
}

Financiamiento: Con aportes de proyecto puente 411-2006 VRAID y Escuela de Medicina, Pontificia Universidad Católica de Chile. 
aun cuando los estudios han mostrado resultados contradictorios ${ }^{3,6,7}$. Por otra parte, se sabe que existen diferencias biológicas en el tamaño del pulmón y de las vías aéreas dependientes del género en individuos normales. Estas diferencias persisten a lo largo de la vida y podrían, en alguna medida, contribuir a una diferente susceptibilidad a desarrollar EPOC en mujeres y hombres ${ }^{8}$.

Dado que en nuestro país no existe información en relación al género de los pacientes con EPOC y a que el porcentaje de la población femenina que fuma alcanza niveles prácticamente iguales a los de los hombres ${ }^{9}$, el objetivo del presente estudio fue evaluar si existen diferencias en las características clínicas y la gravedad de la EPOC dependientes del género en un grupo de 95 pacientes consecutivos con esta enfermedad en que un $40 \%$ corresponde a mujeres.

\section{Material y Métodos}

\section{Pacientes}

Se estudió 95 pacientes que cumplían los criterios diagnósticos de EPOC de acuerdo a $\mathrm{GOLD}^{10}$, que habían dejado de fumar por lo menos 6 meses antes de ser reclutados en forma consecutiva entre Noviembre 2006 y Agosto del 2007 para un estudio de seguimiento de exacerbaciones en el Departamento de Enfermedades Respiratorias de la Universidad Católica de Chile. El protocolo fue aprobado por el comité de ética de nuestra institución y todos los pacientes firmaron un consentimiento informado.

Al ingreso se registró la historia de tabaquismo, expresada en paquetes-año, la edad de comienzo y el tiempo de abandono del tabaquismo, la exposición al humo de biomasa y a contaminantes laborales, y la presencia de comorbilidades. Se registró el nivel educacional y el número de exacerbaciones en el año previo al ingreso. Se midió el peso y la talla y se calculó el índice de masa corporal (IMC). Las características demográficas de los pacientes y su gravedad de acuerdo a los criterios de clasificación de GOLD ${ }^{10}$ se describen en la Tabla 1.

\section{Mediciones}

Función pulmonar. Las mediciones espirométricas se realizaron siguiendo los criterios de las Sociedades Americana de Tórax (ATS) y Europea de Enfermedades Respiratorias (ERS) ${ }^{11}$, utilizando un espirómetro Spiro Analizer ST250 (Fukuda Sangyo, Japón). Se midió la CVF, $\mathrm{VEF}_{1}$ y la relación $\mathrm{VEF}_{1} / \mathrm{CVF}$ antes y después de la administración de $200 \mu \mathrm{g}$ de salbutamol. Para el cálculo de los valores teóricos se empleó los valores de referencia de Hankinson y colaboradores $^{12}$. La hiperinflación pulmonar se evaluó a través de la medición de la capacidad inspiratoria (CI) durante la maniobra de capacidad vital lenta (CVL) y se expresó como porcentaje de los valores de referencia de Lisboa y colaboradores $^{13}$.

Capacidad de ejercicio. La capacidad de ejercicio se midió con la prueba de C6M (six minute walking distance test $6 M W D$ de la literatura inglesa), siguiendo las normas de la $\mathrm{ATS}^{14}$. Para los resultados se empleó el valor más alto de dos pruebas realizadas con un intervalo de al menos 30 minutos. Los valores se expresaron en porcentaje del valor teórico utilizando los valores de referencia de Troosters y colaboradores ${ }^{15}$.

Evaluación clínica. La magnitud de la disnea se evaluó con la escala modificada del Medical Research Council del Reino Unido (MMRC) que tiene una puntuación de 0 a 4 , siendo este último el de mayor disnea ${ }^{16}$.

Para evaluar el nivel clínico de gravedad se calculó el índice multidimensional BODE de gravedad de la EPOC $^{17}$, que incluye el IMC, el grado de obstrucción bronquial evaluado con el $\mathrm{VEF}_{1}$, la magnitud de la disnea medida con el MMRC, y la capacidad de ejercicio, medida con

Tabla 1. Características de los 95 pacientes con enfermedad pulmonar obstructiva crónica

\begin{tabular}{ll}
\hline Variable & $\mathbf{x} \pm \mathbf{D E}$ \\
\hline Género (hombres/mujeres) & $57 / 38$ \\
Edad (años) & $68,6 \pm 7,3$ \\
Tabaquismo (paquetes-año) & $41,7 \pm 21$ \\
Estado GOLD & $\mathrm{n}(\%)$ \\
$\quad$ Leve & $8(8,5)$ \\
Moderada & $28(29,5)$ \\
Grave & $40(42)$ \\
$\quad$ Muy grave & $19(20)$ \\
VEF $\%$ teórico & $47,5 \pm 19,7$ \\
CI \% teórico & $69 \pm 17$ \\
Disnea MMRC & $2,1 \pm 0,7$ \\
C6M \% teórico & $83,5 \pm 25$ \\
Índice de Charlson & $3,63 \pm 1,48$ \\
Índice BODE & $3,32 \pm 2,08$ \\
\hline
\end{tabular}

CI: capacidad inspiratoria; C6M: caminata en 6 minutos; BODE: índice multidimensional de gravedad de EPOC (ver texto). 
la prueba de C6M. El índice BODE tiene una gradación de 0 a 10 puntos, correspondiendo este último al nivel de mayor gravedad.

Calidad de vida. La evaluación del compromiso de la calidad de vida se realizó con las versiones validadas en español de 2 cuestionarios específicos para enfermedades de la vía aérea: el cuestionario de Saint George (SGRQ) ${ }^{18}$ y el cuestionario de enfermedades respiratorias crónicas (chronic respiratory questionnaire; CRQ $)^{19}$. El primero considera 3 aspectos: síntomas, actividades e impacto de la enfermedad y tiene un puntaje de 0 a 100 puntos, correspondiendo el 100 a la peor condición. El CRQ incluye 4 dominios o componentes: disnea, fatiga, control de la enfermedad y aspecto emocional, tiene un puntaje para cada ítem y para el total que se expresa de 1 a 7 , como también de 1 a 140 puntos, siendo el valor máximo la mejor calidad de vida.

Exposición al humo de biomasa y a contaminantes laborales. Para determinar la posible exposición domiciliaria se preguntó a todos los pacientes si habían estado expuestos a inhalación de humo de leña o carbón a lo largo de su vida y si tal exposición había sido preferentemente en la infancia/adolescencia y/o en la vida adulta. La exposición laboral se exploró empleando una encuesta simple similar. No fue posible cuantificar el tiempo ni la magnitud de la exposición a partir de la información proporcionada por los pacientes.

Comorbilidades. Las enfermedades asociadas o comorbilidades se calificaron empleando el índice de Charlson ${ }^{20}$.

\section{Análisis estadístico}

Las variables de distribución normal se expresan como promedio \pm 1 desviación estándar. Las comparaciones entre ambos sexos se realizaron mediante la prueba de $\mathrm{t}$ de Student para muestras independientes. Se empleó la prueba de $\chi^{2}$ para la comparación de variables categóricas. Se consideró significativo un valor de $\mathrm{p}<0,05$.

\section{Resultados}

La Tabla 1 resume las principales características del grupo estudiado. Además de una historia de tabaquismo importante, se aprecia una distribución hacia estados más avanzados de la enfermedad, ya que sólo un $8,5 \%$ tiene una EPOC leve. Asimismo, se observó un grado de escolaridad promedio de 9,3 \pm 4 años, que correspondería a una educación media incompleta. El tiempo de cese del tabaquismo fue de $11 \pm 8$ años. Cuarenta y cuatro de los 95 pacientes refirió exposición a otros contaminantes (combustión de biomasa y laborales). Sólo 12 pacientes recibían oxigenoterapia domiciliaria.

En la Tabla 2 se comparan las características de la enfermedad según género. Se puede observar que para un compromiso funcional similar entre ambos sexos en términos de $\mathrm{VEF}_{1}, \mathrm{CI}$, disnea, capacidad de ejercicio y BODE, el consumo de tabaco fue significativamente menor en las mujeres. Por otro lado, mientras que un número mayor de mujeres que hombres relató exposición a combustión de biomasa (17 versus 8), lo opuesto se observó en la exposición a

Tabla 2. Características de acuerdo al género en los 95 pacientes con enfermedad pulmonar obstructiva crónica

\begin{tabular}{lccc}
\hline & Hombres $(\mathbf{n}=\mathbf{5 7})$ & Mujeres $(\mathbf{n}=\mathbf{3 8})$ & $\mathbf{p}$ \\
\hline Edad (años) & $69,2 \pm 6,8$ & $67,6 \pm 7,9$ & 0,301 \\
Escolaridad (años) & $9,1 \pm 4,2$ & $9,7 \pm 3,8$ & 0,479 \\
IMC (kg×m $\left.{ }^{-2}\right)$ & $25,8 \pm 3,7$ & $27,4 \pm 5$ & 0,095 \\
Tabaquismo (paq-año) & $45,7 \pm 21$ & $35,5 \pm 19,4$ & 0,02 \\
VEF \% teórico & $46,3 \pm 21$ & $49 \pm 17$ & 0,624 \\
CI \% teórico & $70 \pm 19$ & $67 \pm 16$ & 0,447 \\
Disnea MMRC & $2,15 \pm 0,75$ & $2,05 \pm 0,73$ & 0,501 \\
C6M \% teórico & $82 \pm 24$ & $86 \pm 25$ & 0,456 \\
Índice de Charlson & $3,54 \pm 1,36$ & $3,76 \pm 1,65$ & 0,482 \\
BODE puntos & $3,4 \pm 2,0$ & $3,2 \pm 2$ & 0,734 \\
\hline
\end{tabular}

paq-año: paquetes-años; CI: capacidad inspiratoria; C6M: caminata en 6 minutos; BODE: índice multidimensional de gravedad de EPOC (ver texto). 
Tabla 3. Calidad de vida según género en 95 pacientes con enfermedad pulmonar obstructiva crónica

\begin{tabular}{lccc}
\hline & Hombres $(\mathbf{n}=\mathbf{5 7})$ & Mujeres $(\mathbf{n}=\mathbf{3 8})$ & $\mathbf{p}$ \\
\hline Síntomas SGRQ & $47,9 \pm 21,1$ & $44,5 \pm 20,7$ & 0,448 \\
Actividad SGRQ & $71,8 \pm 22,7$ & $71,8 \pm 20,2$ & 1,000 \\
Impacto SGRQ & $47,2 \pm 22,7$ & $47,0 \pm 21,6$ & 0,964 \\
Total SGRQ & $54,9 \pm 19,9$ & $54,5 \pm 17,7$ & 0,922 \\
Disnea CRQ & $18,1 \pm 9,2$ & $21,6 \pm 8,6$ & 0,067 \\
Fatiga CRQ & $15,2 \pm 6,9$ & $15,4 \pm 5,2$ & 0,874 \\
Control CRQ & $15,8 \pm 7,5$ & $17,5 \pm 6,3$ & 0,257 \\
Emocional CRQ & $26,6 \pm 12,6$ & $27,3 \pm 7,7$ & 0,778 \\
Total CRQ & $75,8 \pm 34,5$ & $81,9 \pm 24,1$ & 0,352 \\
\hline
\end{tabular}

SGRQ: Saint George’s Respiratory Questionannaire; CRQ: Chronic Respiratory Questionnaire.

contaminantes laborales (3 versus 16). Las diferencias fueron significativas $(\mathrm{p}=0,001)$.

No hubo diferencias entre ambos sexos en la distribución de la gravedad de la EPOC, en el nivel de escolaridad, en el uso de $\mathrm{O}_{2}$ domiciliario, en las comorbilidades, como tampoco en el número de exacerbaciones en el año previo.

La Tabla 3 muestra los valores totales y de los componentes de los cuestionarios SGRQ y CRQ de acuerdo al género. Los valores no difieren según género, con la excepción del componente disnea del CRQ, que tiende a estar más afectado en los hombres.

\section{Discusión}

Los principales hallazgos del presente estudio realizado en un grupo de pacientes ingresados consecutivamente son: 1) la gravedad de la EPOC evaluada a través de índices funcionales, clínicos y de calidad de vida no demostró diferencias significativas de acuerdo al género; y 2) esta gravedad similar de la EPOC se produjo con una historia de tabaquismo significativamente inferior en las mujeres.

En los últimos años se ha demostrado que la EPOC ha aumentado en las mujeres en varios países $^{21}$, lo que se ha relacionado al aumento del tabaquismo en ellas. Sin embargo, no se ha logrado establecer si las mujeres son más susceptibles a desarrollar la enfermedad, existiendo evidencias contradictorias ${ }^{2,6,21,22}$. Por otra parte, también es discordante la información respecto a la mortalidad según género ${ }^{23,24}$, pues si bien parecería mayor en hombres, cuando se estandariza con la mortalidad general de acuerdo al género resulta mayor en las mujeres.
De acuerdo a la información disponible, éste es el primer estudio en Chile que evalúa la gravedad de la EPOC según género. En relación a otros estudios sobre la susceptibilidad de las mujeres fumadoras a desarrollar EPOC, definida como igual gravedad en términos de $\mathrm{VEF}_{1}$ en relación a la magnitud del tabaquismo, nuestros resultados concuerdan con los comunicados por varios autores ${ }^{7,25,26}$. El reciente estudio de Martínez y colaboradores ${ }^{25}$, analizó las características de los pacientes sometidos a cirugía de reducción de volumen pulmonar. Estos autores observaron que, a igual gravedad de la EPOC según GOLD, las mujeres tenían una historia de tabaquismo significativamente inferior que la de los hombres. A diferencia de nuestro estudio, sus pacientes tenían una enfermedad más avanzada y las mujeres reportaron mayor disnea y mayores síntomas de depresión. En el presente estudio, la disnea fue semejante entre hombres y mujeres tanto de acuerdo a la escala MMRC como a la escala de Borg y, contrariamente a lo comunicado por Martínez y cols ${ }^{25}$, los hombres presentaron una tendencia a una mayor disnea en el cuestionario de calidad de vida CRQ. Machado y colaboradores ${ }^{7}$ en su estudio de seguimiento de pacientes en oxigenoterapia domiciliaria también comunicaron menor consumo de tabaco en las mujeres para igual $\mathrm{VEF}_{1}$ y un mayor riesgo de muerte que los hombres. De Torres y colaboradores ${ }^{27}$ compararon 53 hombres y 53 mujeres consecutivos pareados por $\mathrm{VEF}_{1}$. Al igual que en los estudios anteriores, observaron un menor consumo de tabaco en las mujeres, quienes presentaron además un peor intercambio de gases, menor C6M, mayor disnea y una peor calidad de vida. Nuestros resultados sólo coinciden en el menor consumo de 
tabaco, pues no observamos diferencias en la gravedad de la enfermedad entre ambos sexos. No podemos descartar que esto último pueda deberse a un error tipo beta, debido al número relativamente reducido de mujeres incluido en nuestro estudio.

Una diferencia importante en la presentación estructural de la EPOC ha sido recientemente comunicada en dos estudios ${ }^{25,28}$. Dansfield y colaboradores ${ }^{28}$ evaluaron la gravedad del enfisema en la tomografía axial computada (TAC) en pacientes con EPOC clasificados de acuerdo a GOLD. Observaron que para cada nivel de gravedad, los hombres tenían más enfisema que las mujeres. Los resultados de Martínez y cols ${ }^{25}$, también muestran menos enfisema en la TAC en las mujeres, el que a su vez es preferentemente central, y en un subgrupo en que se analizó además la histología, se apreció que las mujeres tenían mayor compromiso de la vía aérea periférica, que presentaba menor diámetro y paredes desproporcionadamente engrosadas. Como estas alteraciones estructurales se asociaron a un menor consumo de tabaco en las mujeres, podrían indicar una mayor susceptibilidad del género femenino a desarrollar EPOC con afectación preferente de la vía aérea. Aunque los mecanismos involucrados se desconocen, en parte pueden explicarse por el menor tamaño del pulmón y la vía aérea de las mujeres ${ }^{8}$. Otro factor que podría explicar esta mayor susceptibilidad de las mujeres en nuestro estudio es el antecedente la exposición a combustión de biomasa. La mayoría de nuestras pacientes sufrieron dicha exposición en la infancia, en sus domicilios, en cocinas cerradas con combustión de leña y/o con calefacción empleando carbón como combustible. Excepcionalmente este último tipo de exposición continuaba en el momento de la inclusión en el estudio.

Una de las limitaciones de nuestro estudio es la imposibilidad de cuantificar la magnitud de la exposición a combustión de biomasa, que nos hubiera permitido establecer su contribución a desarrollar o agravar la EPOC en las mujeres. Por otro lado, como ya se mencionó, la ausencia de diferencias en la gravedad de la enfermedad entre ambos sexos puede deberse al número relativamente reducido de mujeres, que aumenta el riesgo de un error tipo beta. Una limitación adicional es que al haber incluido sólo a ex fumadores, participantes de un estudio de seguimiento, esta muestra puede no ser representativa de la población general de pacientes con dicha enfermedad.

En resumen, nuestros resultados muestran que las mujeres del grupo estudiado presentan una gravedad semejante a la de los hombres, aunque con una historia de tabaquismo muy inferior. La evaluación de gravedad empleada, a diferencia de la mayoría de los estudios al respecto, no sólo incluyó la gravedad según GOLD, sino que también la hiperinflación pulmonar, el índice BODE, las comorbilidades y la calidad de vida. Un aspecto de interés será evaluar en el futuro la progresión de la EPOC según género, empleando este espectro amplio de evaluación de la gravedad para establecer con mayor propiedad si la enfermedad es más grave en las mujeres, como lo plantea su aumento de la mortalidad, de manera de centrar en ellas las medidas para disminuir el tabaquismo.

\section{Agradecimientos}

Nuestros agradecimientos a las enfermeras señoras Fedora Arancibia R. y Alicia Leiva G. por su importante apoyo técnico.

\section{Bibliografía}

1.- VIEGI G, SCOGNAMilio A, BALDACCI S, PISTELLI F, CARROZZI L. Epidemiology of chronic obstructive pulmonary disease. Respiration 2001; 68: 4-19.

2.- MANNINO D M, HOMA D M, AKINBAMI L J, FORD E S, REDD S C. Chronic obstructive pulmonary disease surveillance-United States 1971-2000. Respiratory Care 2002; 47: 1184-99.

3.- CHAPMAN K R, TASHKIN D P, PYE D J. Gender bias in the diagnosis of COPD. Chest 2001; 119: 16915.

4.- SORIANO J B, MAIER W C, EGGER P, VISICK G, THAKRAR B, SYKES J, et al. Recent trends in physician diagnosed COPD in women and men in the UK. Thorax 2000; 55: 789-94.

5.- DEMEO D L. The Yin and Yang of COPD. Sex/ gender differences in the national emphysema treatment trial. Editorial. Am J Respir Crit Care Med 2007; 176: 222-3

6.- SILVERMAN E K, WEISS S T, DRAZEN J M, CHAPMAN H A, CAREY V, CAMPBELL E J, et al. Gender-related differences in severe early-onset chronic obstructive pulmonary disease. Am J Respir Crit Care Med 2000; 162: 2152-8.

7.- MACHADO M C, KRISHNAN J A, BUIST S A, BILDERBACK A L, FAZOLO G P, SANTAROSA M $G$, et al. Sex differences in survival of oxygendependent patients with chronic obstructive pulmonary disease. Am J Respir Crit Care Med 2006; 174: 524-9.

8.- BECKLAKE M R, KAUFFMANN F. Gender differences in airway behavior over the human life span. Thorax 1999; 54:1119-38.

9.- MENEZES A M, PÉREZ-PADILLA R, JARDIM J R, MUIÑO A, LÓPEZ M V, VALDIVIA G, et al. Chronic 
obstructive pulmonary disease in five Latin American cities (the PLATINO study): a prevalence study. Lancet 2005, 366: 1875-81.

10.- RABE K F, HURD S, ANZUETO A, BARNES P J, BUIST S A, CALVERLEY P, et al. Global strategy for the diagnosis, management, and prevention of chronic obstructive pulmonary disease. GOLD executive summary. Am J Respir Crit Care Med 2007; 176: 53255.

11.- MILLER M R, HANKINSON J, BRUSASCO V, BURGOS F, CASABURI R, COATES A, et al. Standardisation of spirometry. Eur Respir J 2005; 26 : 319-38

12.- HANKINSON J L, ODENCRANTZ J R, FENDAN K B. Spirometric reference values from a sample of the general US population. Am J Respir Crit Care Med 1999; 159: 179-87.

13.- LISBOA C, LEIVA A, PINOCHET R, REPETTO P, BORZONE G, DÍAZ O. Valores de referencia de la capacidad inspiratoria en sujetos sanos no fumadores mayores de 50 años. Arch Bronconeumol 2007; 43: 485-9.

14.- AMERICAN THORACIC SOCIETY STATEMENT. Guidelines for the six-minute walk test. Am J Respir Crit Care Med 2002; 166: 111-7.

15.- TROOSTERS T, GOSSELINK S, DECRAMER M. Six minute walking distance in healthy elderly subjects. Eur Respir J 1999; 14: 270-4.

16.- MAHLER D A, WELLS C K. Evaluation of clinical methods for rating dyspnea. Chest 1988; 93: 580-6.

17.- CELLI B R, COTE C G, MARÍN J M, CASANOVA C, MONTES DE OCA M, MÉNDEZ R A, et al. The body-mass index, airflow obstruction, dyspnea and exercise capacity index in chronic obstructive pulmonary disease. N Engl J Med 2004; 350: 100512.

18.- JONES P W, QUIRK F H, BAVEYSTOCK C M, LITTLEJOHNS P. A self complete measure of health status for chronic airflow limitation: the St George's Respiratory Questionnaire. Am Rev Respir Dis 1992; 145: $1321-7$.
19.- GÜELL R, CASAN P, SANGENÍS M, SANTÍS J, MORANTE F, BORRAS J M, et al. Traducción española y validación de un cuestionario de calidad de vida en pacientes con enfermedad pulmonar obstructiva crónica (EPOC). Arch Bronconeumol 1995; 31: 20210.

20.- CHARLSON M, SZATROWSKI T P, PETERSON J, GOLD J. Validation of a comorbidity index. J Clin Epidemiol 1994; 47: 1245-51.

21.- MANNINO D M. Women and chronic obstructive pulmonary disease. Does Sex influence survival? Editorial. Am J Respir Crit Care Med 2006; 174: 488-9.

22.- DE TORRES J P, CAMPO A, CASANOVA C, AGUIRRE-JAIME A, ZULUETA J. Gender and chronic obstructive pulmonary disease in high-risk smokers. Respiration 2006; 73: 306-10.

23.- RINGBAEK T, SEERSHOLM N, VISKUM K. Standardised mortality rates in females and males with COPD and asthma. Eur Respir J 2005; 25: 891-5.

24.- SUNYER J, ANTÓ J M, MCFARLANE D, DOMINGO A, TOBÍAS A BARCELÓ M A, et al. Sex differences in mortality of people who visited emergency rooms for asthma and chronic obstructive pulmonary disease. Am J Respir Crit Care Med 1998; 158: 851-6.

25.- MARTÍNEZ F J, CURTIS J L, SCIURBA F, MUMFORD J, GIARDINO N D, WEIMANN G, et al. Sex differences in severe pulmonary emphysema. Am J Respir Crit Care Med 2007; 176: 243-52.

26.- WATSON L, SCHOUTEN J P, LÖFDAHL C G, PRIDE N B, LAITINEN L A, POSTMA D S, et al. Predictors of COPD symptoms: does sex of the patient matter? Eur Respir J 2006; 28: 311-8.

27.- DE TORRES J P, CASANOVA C, HERNÁNDEZ C, ABREU J, AGUIRRE-JAIME A, CELLI B R. Gender and COPD in patients attending a pulmonary clinic. Chest 2005; 128: 2012-6.

28.- DRANSFIELD M T, WASHKO G R, FOREMAN M G, SAN JOSÉ ESTEPAR R, REILLY J, BAILEY W C. Gender differences in the severity of CT emphysema in COPD. Chest 2007; 132: 464-70.

Correspondencia a:

Dra. Carmen Lisboa B.

Departamento de Enfermedades Respiratorias

Pontificia Universidad Católica de Chile

Marcoleta 352, Primer piso.

E-mail: clisboa@med.puc.cl 